\title{
Making the most of context-awareness in brain-computer interfaces
}

\author{
Sareh Saeedi, Tom Carlson, Ricardo Chavarriaga, José del R. Millán \\ Chair in Non-Invasive Brain-Machine Interface (CNBI), \\ Center for Neuroprosthetics, School of Engineering, EPFL \\ Lausanne, Switzerland \\ sareh.saeedi@epfl.ch
}

\begin{abstract}
In order for brain-computer interfaces (BCIs) to be used reliably for extended periods of time, they must be able to adapt to the users evolving needs. This adaptation should not only be a function of the environmental (external) context, but should also consider the internal context, such as cognitive states and brain signal reliability. In this work, we propose three different shared control frameworks that have been used for BCI applications: contextual fusion, contextual gating, and contextual regulation. We review recently published results in the light of these three context-awareness frameworks. Then, we discuss important issues to consider when designing a shared controller for BCI.
\end{abstract}

Index Terms-brain-computer interface (BCI), shared control, adaptive assistance

\section{INTRODUCTION}

Brain-Computer Interfaces (BCIs) enable people with severe motor disabilities to restore (some of) their communication and control capabilities [1]. The BCI monitors the user's brain activity and translates his/her intentions into commands, which are sent to an external device, such as a wheelchair or a prosthetic device [2], [3]. In this framework, there are several challenges for determining the subject's intention using an uncertain channel, such as BCI. These challenges are mostly associated with relatively low accuracies, low temporal precision, low information transfer rates, measurement noise, and uncertainty in the system. There are several sources of uncertainty in the system, such as the user's internal state (attention, etc.), non stationarity of the brain signal, variation of the class discriminative information within and between users [4].

In this respect, the use of shared control systems, in which both the user and the system contribute to the control process is beneficial [5], [6]. Using shared control techniques for BCI results in better performance, higher speed and safety while reducing the required effort [4], [7], [8]. However, given the mentioned challenges, there are critical issues to be considered in designing a shared control system for BCI applications. Some of these issues are mentioned in the following:

(i) Assistive robots, like wheelchairs, neural prostheses and telepresence robots might work with different levels of control. Four major types of control can be distinguished depending on the users involvement in the control task (Figure 1). Manual control means the robot is controlled directly by the user's commands. Shared control systems make use of the com- mands from both the user and the device. In contrast, SemiAutonomous control relies on the robot being autonomous for a time interval and requires the users input to define the next goal. Autonomous control means that once the robot is started, it will operate compltely independatnly and will not require any user input [5]. An important aspect is the level of shared control in the system or the level of assistance that the system provides for the user. The users of such devices often prefer to have authority over the device rather than to be controlled by it. In other words, the system should provide a transparent assistance for the user [4], [9], [10].

(ii) In order to design a shared control system, commands from the BCI and the contextual information should be fused to determine the final command to the device. Therefore, defining methods to fuse these sources of information is of great importance.

(iii) BCI systems can benefit from either a continuous or a discrete shared control system. The former usually requires constant involvement and therefore results in high workload for the user. The latter, however, might be slower in performing the desired task [11]. The choice of each of the mentioned paradigms depends on the system and the application goals.

(iv) Shared control techniques usually have predefined settings based on the task and the environment in which the task is performed. In addition, the level of assistance they provide is usually constant over time. However, in order to have an effective control, this level should be adaptive so as to complement the user's capabilities which change over time. In this way, the user always remains in control of the brain actuated device [10].

(v) Shared or hybrid control can be used to combat the fatigue associated with a particular control channel or level of control. This allows the users to take over the control when they want to do so [4], [12].

The main goal of this work is to study the different approaches of combining internal and external context in shared control BCI systems. First, we propose three different frameworks for combining internal and external context. We, also, review previously presented results in the light of these three context-awareness frameworks. Then, we discuss important issues to consider when designing a shared controller for BCI. 


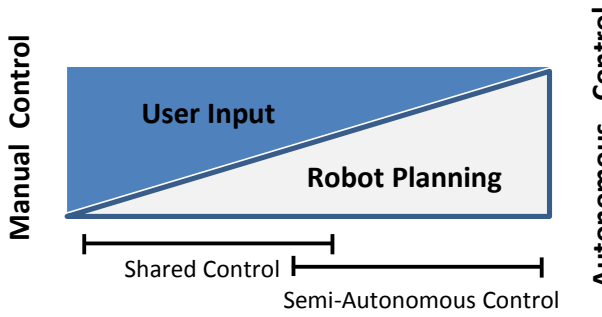

Fig. 1. Different levels of control for assistive devices

\section{APPROACHES}

Brain-controlled assistive technology, such as wheelchairs, telepresence robots and neuroprostheses offer promising solutions to the problems suffered by people with severe motor disabilities. However, in order to reliably operate such devices using a $\mathrm{BCI}$ in real-world environments, some degree of assistance (shared control) is required to compensate for the relatively low information transfer rate and performance variability of the BCI. The level of this assistance is usually only a function of the external context (i.e. the surrounding environment) [13]. However each person has different needs and abilities, which in turn change over time. We refer to this as the internal context, which encompasses cognitive states and changes in the users brain patterns (Figure 2).

Therefore, many studies have investigated how to capture the instantaneous needs of user's, such that the level of assistance could be adjusted accordingly. To provide shared control for BCI, different approaches have been taken that can be summarized in mainly three different categories (Figure 3): (i)contextual fusion, where the internal and the external context contribute directly to the final control command, (ii)contextual gating, in which an initial command can be confirmed or refused by an internal or an external context, and (iii)contextual regulation, where an internal or an external context can refine the controller.

\section{A. Contextual fusion}

In this framework, both the internal and the external context contribute directly to the final control command (Figure 3(a)). Different techniques can be used for fusing the internal and external context in shared control systems. All the behaviors (like obstacle avoidance, or goal-directed behaviors) assumed for a task should be merged together according to a specific rule. Competitive methods, weighted sums of different behaviors, and probabilistic reasoning are among the implemented rules for this purpose.

An example of such a paradigm is controlling a telepresence robot using brain signals [8], [14]. While the user is responsible for high level commands, such as going to the right or the left, the device is responsible for low level commands, including obstacle avoidance. The users learn to voluntarily modulate electroencephalogram (EEG) oscillatory rhythms by executing two motor imagery tasks. Each of these mental commands is associated with a steering command. In case of no command from the user, the robot should move forward. This provides

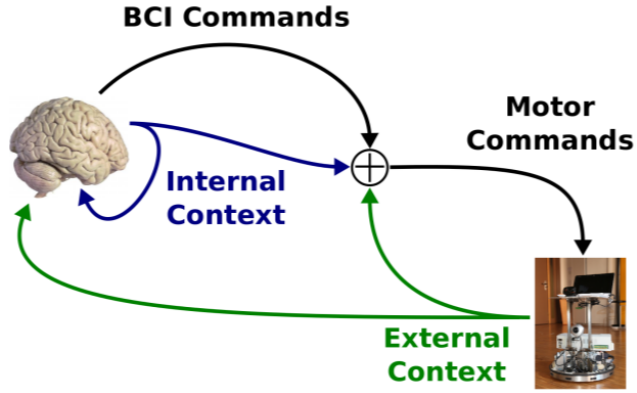

Fig. 2. Proposed context-aware adaptive shared control architecture for BCI.

the internal context for the shared control system, which in this case, represents the user's intent. On the other side, the robot is equipped with infrared sensors, capable of detecting obstacles up to $30 \mathrm{~cm}$. In this way, the external context is provided for the shared control system. Without shared control, the robot stops in front of an obstacle and waits for the user's next command. However, if shared control is enabled, the robot moves around the obstacle, until the path is free.

A study involving patients controlling a telepresence robot reveals that when a BCI is combined with shared control (using both internal and external context), users can achieve a similar level of performance as is attained with a manual (2-button), no shared control condition. Also, patients are able to attain a comparable level of performance to healthy subjects [14]. This is illustrated in Figure 4 as the ratio of the time required and the ratio of the number of commands required when using $\mathrm{BCI}$ and when using the manual input. This also supports the initial results for healthy subjects [8]. In general, the contextawareness framework is beneficial for both the patients and the healthy subjects.

Another study, which implements a "fusion" framework for driving a wheelchair, enables the user to dock to an obstacle [15]. A discrete two-button input device is used to represent the output of a two-class asynchronous BCI (turn to the right or turn to the left). This serves as the internal context for the shared control system. Also, the shared controller benefits from two sources of external context: the location of the potential targets that the user may wish to reach and the location of the obstacles to avoid. For the former to be achieved, any obstacle is considered to be a target, provided that it is located directly in front of the wheelchair. Two approaches to shared control have been taken and compared for this experiment: the reactive shared control, where the shared controller only reacts to the user's input signals, e.g. by refining exactly how far and fast to make a turn and the proactive shared control, where the controller will proactively turn to avoid obstacles, and align to the narrow doorways, even before receiving input from the user.

Overall performance of navigating with a wheelchair in a cluttered office environment has been compared using reactive and proactive shared control [15]. Figure 5 shows a typical trajectory driven in the experiment. The crosses indicate delivering a turning command by the subject. If there is not 
(a) Fusion

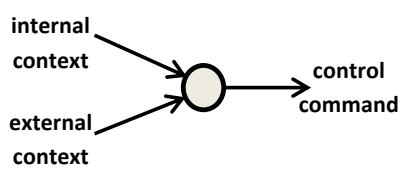

(b) Gating

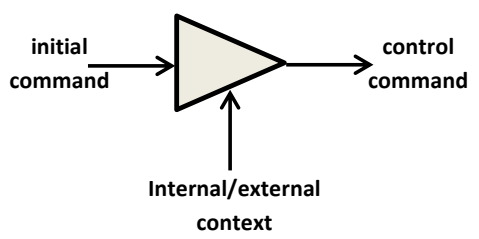

(c) Regulation

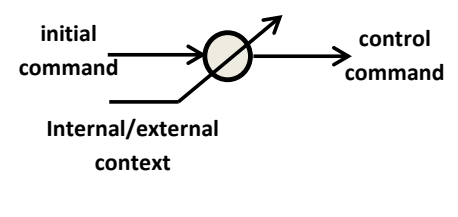

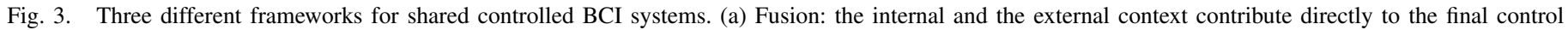

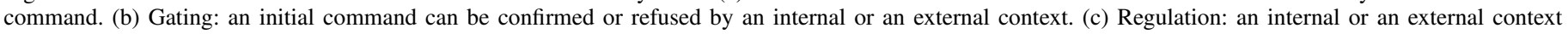
can refine the controller.

corresponding cross, the command is a proactive one.

Different metrics have been used for this comparison. A very significant improvement in task completion time was observed in proactive shared control compared to the reactive shared control. The average wheelchair speed was found to be significantly higher and a significantly reduced number of stops was observed using the proactive shared control. Also, the number of commands issued by the user decreased significantly to two-thirds. In general, in the cluttered environment, the users preferred to use the proactive shared control.

The two studies mentioned here use the information about the environment as the external context. However, any other source of information, which is not internal to the user, but could help them to accomplish the task can also serve as the external cue. For instance, a language model has been implemented in a hybrid-BCI text entry system, which makes use of the same asynchronous two-class BCI system [16]. All the available characters are shown to the subject using underlying inference mechanisms based on a Hu-Tucker binary tree, which ensures the minimum average number of commands to reach a character based on a prefix-based language model. This approach reduces the user's mental workload required for performing the task. The control system is also enhanced with an error-handling mechanism, in which electromyography (EMG) monitoring allows the user to "undo" the latest BCI action. Therefore, for the shared control system, both EEG and EMG signals are used as the internal context while the language model provides the external context in the "fusion" framework.

\section{B. Contextual gating}

In this framework, the initial signal serves as a suggested control command and can be confirmed or refused by querying internal or external contextual information (Figure 3(b)). In other words, the initial signal, which itself can be derived from either a fusion or regulation framework (see next section), should be confirmed by the internal or external commands before being executed as the control command.

A semi-autonomous navigation strategy has been introduced for a mobile robot which follows the gating framework for shared control [17]. In a navigation task, there could be cases where two actions have the same probability to be executed according to the robot analysis. Also, the robot could have a wrong belief about an action, for instance, docking to an obstacle. In such situations, an interaction between the subject and the robot is required. In this respect, an interface that allows the user to provide an answer to that proposition would be required.

In this study, visual feedback has been used to show the proposed action and the possibility of using error-related potentials (ErrP) has been investigated. First, the robotic system analyzes the environment using its laser scanner so as to determine the possible places of human-robot interaction (e.g. crossings). This serves as the external context for the system. Then, the mobile robot proposes the most probable action to the subject who can confirm or refuse the proposition. This confirmation/refusal can be done by means of a button or a BCI, which makes the internal context.

In [18], two types of brain potentials have been investigated to be used as the confirmation/refusal: contingent negative variation $(\mathrm{CNV})$ as the anticipatory brain potentials and ErrP. Further studies on the use of ErrP as well as CNV potentials in a simulated car driving framework [19], [20] have also confirmed the feasibility of using these EEG signals as the internal context in such paradigms. Yet, real-time detection of such brain potentials needs to be further investigated.

The experiment performed in [18] consists of navigation in a virtual environment. The subject evaluates the system's performance by pressing a key. Figure 6 shows the grand average evoked potential (ERP) on the $\mathrm{Cz}$ electrode between two consecutive crossings. Time $\mathrm{t}=0$ corresponds to the feedback onset at the beginning of a maze segment. After 1 second, the robot starts to move and takes three seconds to reach the next intersection. A feedback-evoked positive inflection can be clearly seen about $200 \mathrm{~ms}$ after the stimulus onset $(\mathrm{t}=0$ and $\mathrm{t}=4 \mathrm{~s}$ ). A slow negative deflection, consistent with the reported CNV appears during the time between the two crossings in the maze (Figure 6, left).

ErrP has been observed in frontocentral areas (electrode Fz). Figure 6 (right) shows the grand average difference ERP (i.e. erroneous minus correct trials) across all subjects. The positive peak of the ErrP appears shortly after 400ms after visual feedback. The same results has been observed when the subjects press keys for indicating their confirmation/refusal. Based on the ErrP classification performance in the ROC (Receiver Operating Characteristic) space, it can be seen that erroneous and correct trials can be classified above random level. The 


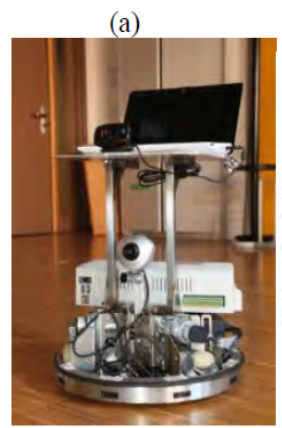

(b)

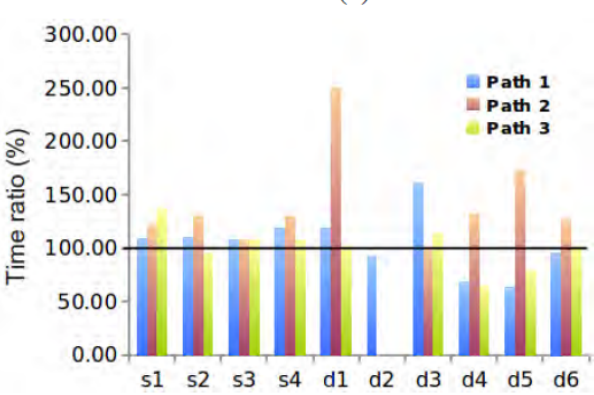

(c)

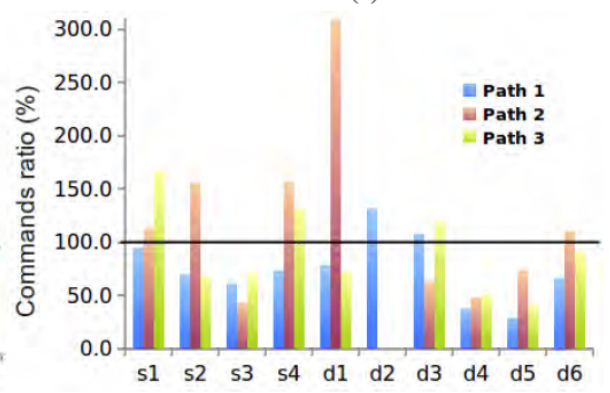

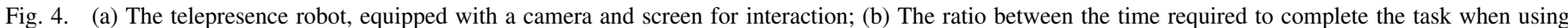

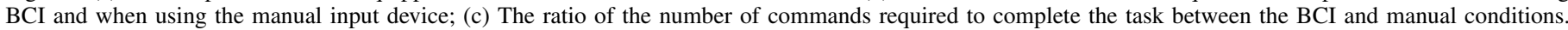
s1-4 are healthy subjects and d1-6 are the motor-disabled end-users [14].

same results have been observed in case of only monitoring the action rather than pressing a key, and using tactile feedback instead of visual. Overall, the results confirm that these brain correlates can be observed in realistic interactive situations.

\section{Contextual regulation}

In this framework, the level of assistance (shared control) can be regulated by means of an internal or an external context, i.e. the parameters of the controller can be adjusted on the fly. Again, the initial signal can be derived from another framework (Figure 3(c)). A semi-autonomous navigation system has been introduced which follows such a regulation framework [21]. This system is also based on proposing actions to the user and waiting for confirmation/refusal from them, as discussed in the previous section. The difference, however, is that the user's habits are learned when navigating in a known environment so as to anticipate the next desired destination, ahead of time in order to reduce the user's workload. For this purpose, a dynamic Bayesian network (DBN) has been used to track the user's intended actions or goal destinations.

A different approach has been taken in [22], which is based on human monitoring of the performance of an external autonomous device. In this experiment, ErrP was found in the EEG while the users received erroneous feedback correlated to the cursor direction of movement (i.e., opposite to the target location). Upon identification of errors, learning of the optimal behavior could be achieved by decreasing the likelihood of repeating such decisions in the same context. In this way, the user provides reinforcement signals that can be used by the system to improve the overall performance.

In addition, some hybrid BCI paradigms can be considered as following the "regulation" framework. A hybrid approach for human computer interaction (HCI) has been proposed in [23], where uses human gestures as the control commands and brain activity is exploited to provide implicit feedback about the recognition of such commands. This can be used afterwards to adapt the behaviour of the HCI system.

Moreover, it would be crucial to assess the capabilities of the user while controlling a BCI system, as it is not usually constant over time. This allows the shared control system to regulate the level of assistance accordingly. We propose to achieve this, by characterizing the reliability of the BCI decoders [24] as an internal context and by using a combination of different online task performance metrics [25] as an external context.

Online estimation of brain signal reliability: The variance in trial length can occur for many reasons, such as changes in attention, fatigue, stress etc. Shared control could compensate for this by, for example, altering the speed or reaction time of a robotic device according to the predicted BCI trial length. In a five subject study, we found that the entropy rate of the EEG signals while subjects control a motor imagery BCI is lower when subjects take a long time to deliver a command. Based on this, we developed a method to predict whether the current command will be emitted quickly or slowly according to the entropy of the first few samples of each trial [24].

The results confirm that we can reliably predict the trial length class (i.e. fast or slow) based on the Entropy of the EEG using a LDA classifier, see Figure 7 [24]. Performance for all subjects (AUC) exceeds 0.7 based on the data samples acquired before the subject median delivery time.

Online task performance metrics: We use online task performance metrics to modulate the level of assistance provided by a shared control system, such that it is well-matched to the users current and ever-evolving needs. These consist of metrics commonly used (post-experiment) to evaluate shared control systems. In this experiment, subjects were instructed to navigate around a complex simulated environment, using their left hand to operate a time-restricted 2-button input, which emulated the output decisions of our motor imagerybased BCI. Periodically, subjects were asked to simultaneously perform a demanding secondary (reaction) task with their right hand, which was designed to increase their workload [25]. During the experiment the assistance modulation factor (AMF) was computed online, based on the number of commands generated by the user, the number of times assistance was required and the effective navigation time.

The assistance modulation factor (AMF) was able to reliably track the users workload, see Figure 8 . The median level of assistance increases signicantly $(p<0.001)$ when the user has to additionally engage in a demanding secondary task. We also see an improvement in overall task performance (e.g. 


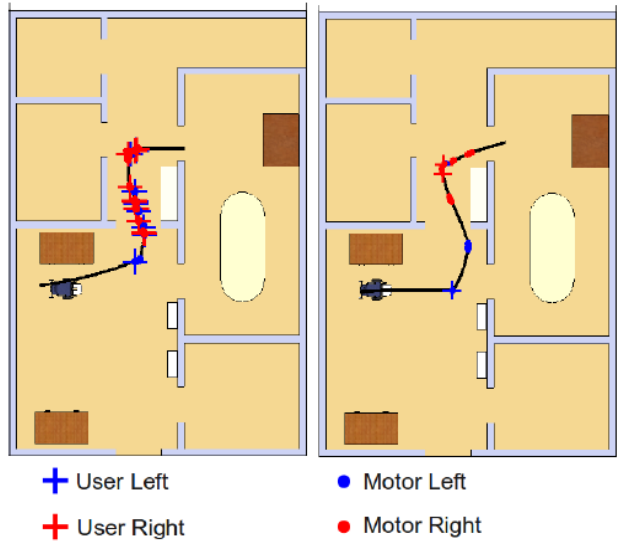

Fig. 5. Commands delivered along typical trajectories that were driven using reactive control (left figure) and proactive control (right figure) in the cluttered environment [15].
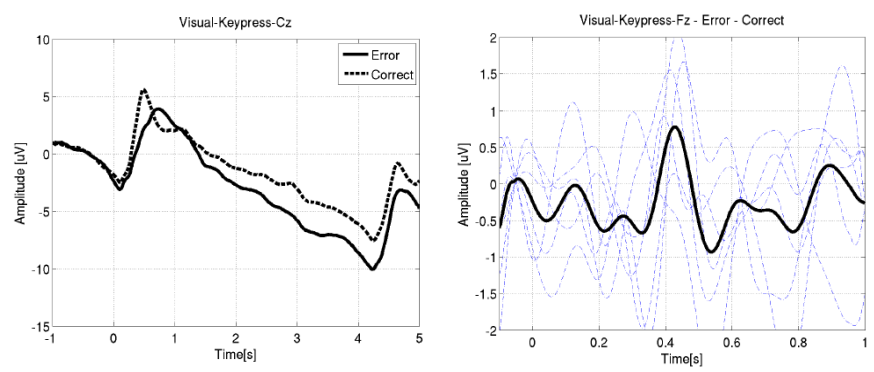

Fig. 6. left: Grand average ERP in $\mathrm{Cz}$ electrode. Visual feedback is presented at time $\mathrm{t}=0$ and $\mathrm{t}=4$ respectively. right: Grand average ERP (error minus correct condition) in Fz electrode. $t=0$ corresponds to the visual feedback onset.

completion time) and a high user-acceptance [25]. Altogether, the AMF provided an online measure of the task difficulty and therefore the amount of assistance required.

\section{DISCUSSION}

Brain-controlled assistive technology, such as neuroprostheses provide communication and control opportunities for people with severe motor disabilities. The ultimate goal of such systems is to perform reliably in real-world environments for extended periods of time. However, an uncertain channel like BCI usually suffers from low temporal precision and information transfer rate. Besides, the non-stationarity of the brain signals, as well as changes of the user's internal state results in variable control capabilities over time. These issues highlight the need to provide some degree of assistance (shared control) for $\mathrm{BCI}$ to compensate for this variability in the $\mathrm{BCI}$ performance.

In the recent literature, several studies are dedicated to using shared control for BCI for increasing the performance, as well as reducing the user's mental workload. It is important to note that the user's cognitive states and therefore, capabilities, which we refer to as the internal context, change over time. However, the main focus of these studies has been to regulate the level of assistance based on the external context (i.e. the surrounding environment).
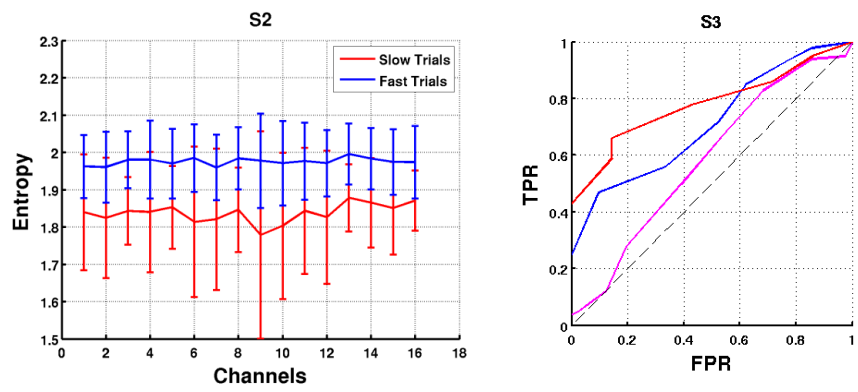

Fig. 7. (a) Average of entropy for fast and slow trials. (b) Classification performance (fast/slow) in ROC curve, when the first $2 \mathrm{~s}$ (magenta), $2.5 \mathrm{~s}$ (black), and 3s (red) of a trial is considered for analysis.

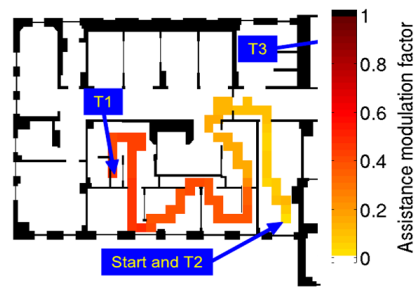

(a)

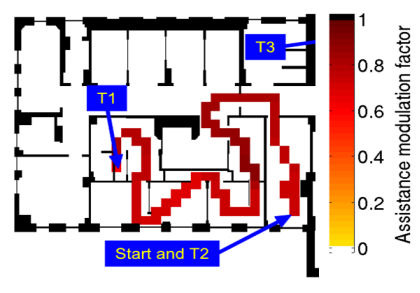

(b)
Fig. 8. Assistance modulation factor when: (a) driving only, (b) driving + secondary task.

In this study, we claim that the shared control approaches presented for BCI, are one or a combination of mainly three different frameworks: contextual fusion, contextual gating, and contextual regulation. Among these frameworks, "fusion" has been commonly used. However, this approach is usually associated with predefined settings based on the task and the environment. Therefore, in order to compensate for variability in the system, other frameworks, such as "gating" or "regulation" have been also used.

Overall, we recommend to consider a couple of issues before designing a shared controller for BCI:

(i) As BCI systems are designed for people with severe motor disabilities, the user's capabilities and therefore the types of signals that can be used as the internal context are important. These signal types and their characteristics (SNR, the level of fatigue they impose,...) are important for defining which framework(s) to be incorporated in the shared control system.

(ii) The potential external context can be defined based on the task and the environment. Moreover, the rate of the required commands and the resolution of the commands depend on the task. These two factors are important in order to define which frameworks to consider. The application goals and the context are of course of great importance for choosing a framework or a combination of them.

(iii) It is crucial to evaluate the price we pay for making mistakes, namely in terms of time and the user's mental workload. If this is relatively high, we suggest to use multiple frameworks (from similar or different types), chained together in a cascading (or parallel) manner in order to provide a reliable regulation of assistance. However, considering many 
parameters in an application may affect the user's learning ability, which is important for long-term use of such systems. Thus, there needs to be a compromise between dynamic regulation of assistance and the complexity when designing a shared control system using different frameworks.

(iv) An important factor to be considered for choosing a shared control framework is the fact that the users often prefer to have authority over the device. In this respect, it helps to benefit from both the internal and external context in order to provide an efficient system.

(v) In case there is a need for adapting the level of assistance continuously, using the regulation framework can be beneficial.

(vi) As in shared control systems different sources of information contribute to the final control command, it is always critical to specify the ones with higher priority. In other words, conflict among different signals should be taken into account and managed in a proper way.

(vii) In order to assess the performance of a shared control system, we need to use a combination of metrics, e.g. rate of commands, time, mental workload, etc. The choice of these metrics and the weight which is attributed to them needs to be further investigated. In other words, further study is required on how to rate the contributions of different actors in a shared control system.

In conclusion, we propose that taking into account both the internal and the external context in designing a shared controller for BCI can enhance their performance over extended periods of time. This can be further investigated by assessing the user acceptance of such systems.

\section{ACKNOWLEDGMENT}

This work is supported by Swiss National Center of Competence in Research (NCCR) Robotics and the European ICT Programme Project TOBI FP7-224631.

\section{REFERENCES}

[1] J. Wolpaw, N. Birbaumer, D. McFarland, G. Pfurtscheller, and T. Vaughan, "Brain-computer interfaces for communication and control." Clin Neurophysiol, vol. 113, no. 6, pp. 767-791, Jun 2002.

[2] J. d. R. Millán, P. W. Ferrez, F. Galán, E. Lew, and R. Chavarriaga, "Non-invasive brain-machine interaction," Int J Pattern Recognition and Artificial Intelligence, vol. 22, no. 5, pp. 959-972, 2008.

[3] J. d. R. Millán, R. Rupp, G. Müller-Putz, R. Murray-Smith, C. Giugliemma, M. Tangermann, C. Vidaurre, F. Cincotti, A. Kübler, R. Leeb, C. Neuper, K. Müller, and D. Mattia, "Combining braincomputer interfaces and assistive technologies: State-of-the-art and challenges," Frontiers in Neuroscience, vol. 4, p. 161, 2010.

[4] B. Allison, S. M. Dunne, R. Leeb, J. d. R. Millán, and A. Nijholt, Towards Practical Brain-computer Interfaces : Bridging the Gap from Research to Real-world Applications, ser. Biological and medical physics, biomedical engineering. Heidelberg: Springer Verlag, 2012.

[5] H. K. Kim, S. J. Biggs, D. W. Schloerb, J. M. Carmena, M. A. Lebedev, M. A. L. Nicolelis, and M. A. Srinivasan, "Continuous shared control for stabilizing reaching and grasping with brain-machine interfaces." IEEE Trans Biomed Eng, vol. 53, no. 6, pp. 1164-1173, Jun 2006.

[6] D. Vanhooydonck, E. Demeester, M. Nuttin, and H. V. Brussel, "Shared control for intelligent wheelchairs: an implicit estimation of the user intention," in Proceedings of the ASER '03 1 st International Workshop on Advances in Service Robotics, pp. 13-15.

[7] F. Galán, M. Nuttin, E. Lew, P. W. Ferrez, G. Vanacker, J. Philips, and J. d. R. Millán, "A brain-actuated wheelchair: Asynchronous and noninvasive brain-computer interfaces for continuous control of robots." Clin Neurophysiol, vol. 119, no. 9, pp. 2159-2169, Sep 2008.
[8] L. Tonin, R. Leeb, M. Tavella, S. Perdikis, and J. d. R. Millán, "The role of shared-control in BCI-based telepresence," in Proc. of 2010 IEEE International Conference on Systems, Man and Cybernetics, 2010.

[9] P. Nisbet, "Who's intelligent? Wheelchair, driver or both?" in Proceedings IEEE International Conference on Control Applications, Glasgow, Scotland, U.K., September 2002.

[10] J. Philips, J. d. R. Millán, G. Vanacker, E. Lew, F. Galán, P. Ferrez, H. V. Brussel, and M. Nuttin, "Adaptive Shared Control of a Brain-Actuated Simulated Wheelchair," in Proceedings of the 10th IEEE International Conference on Rehabilitation Robotics, 2007, pp. 408-414.

[11] X. Perrin, F. Colas, C. Pradalier, R. Siegwart, R. Chavarriaga, and J. del R. Millán, "Learning user habits for semi-autonomous navigation using low throughput interfaces," in SMC. IEEE, 2011, pp. 1-6.

[12] Q. Zeng, B. Rebsamen, E. Burdet, and C. Teo, "A collaborative wheelchair system," Neural Systems and Rehabilitation Engineering, IEEE Transactions on, vol. 16, no. 2, pp. 161-170, 2008.

[13] L. Tonin, T. Carlson, R. Leeb, and J. del R. Millán, "Brain-controlled telepresence robot by motor-disabled people," in Proc. Annual International Conference of the IEEE Engineering in Medicine and Biology Society EMBC 2011, 2011, pp. 4227-4230.

[14] T. Carlson, R. Leeb, G. Monnard, A. Al-Khodairy, and J. d. R. Millán, "Driving a BCI Wheelchair: A Patient Case Study," in Proceedings of TOBI Workshop III: Bringing BCIs to End-Users: Facing the Challenge, 2012, pp. 59-60.

[15] T. Carlson, G. Monnard, R. Leeb, and J. d. R. Millán, "Evaluation of Proportional and Discrete Shared Control Paradigms for Low Resolution User Inputs," in Proceedings of the 2011 IEEE International Conference on Systems, Man, and Cybernetics (IEEE SMC 2011). IEEE, 2011, pp. 1044-1049.

[16] S. Perdikis, A. Ramsey, R. Leeb, J. Williamson, A. Al-Khodairy, R. Murray-Smith, and J. d. R. Millán, "Clinical evaluation of a hybridbci text-entry system," in Proceedings of TOBI Workshop III: Bringing BCIs to End-Users: Facing the Challenge, 2012, pp. 75-76.

[17] X. Perrin, R. Chavarriaga, F. Colas, R. Siegwart, and J. Millán, "Braincoupled interaction for semi-autonomous navigation of an assistive robot." Robotics and Autonomous Systems, vol. 58, no. 12, pp. 1246$1255,2010$.

[18] R. Chavarriaga, X. Perrin, R. Siegwart, and J. d. R. Millán, “Anticipationand error-related EEG signals during realistic human-machine interaction: A study on visual and tactile feedback," in 34th International Conference of the IEEE Engineering in Medicine and Biology Society (EMBC'12), 2012.

[19] H. Zhang, R. Chavarriaga, M. K. Goel, L. A. Gheorghe, and J. d. R. Millán, "Improved Recognition of Error Related Potentials through the use of Brain Connectivity Features," in The 34th Annual International Conference of the Engineering in Medicine and Biology Society, 2012.

[20] Z. Khaliliardali, R. Chavarriaga, L. A. Gheorghe, and J. d. R. Millán, "Detection of Anticipatory Brain Potentials during Car Driving," in Proceeding of the 34th Annual International IEEE EMBS Conference, 2012.

[21] X. Perrin, F. Colas, R. Chavarriaga, C. Pradalier, J. d. R. Millán, and R. Siegwart, "Learning user habits for semi-autonomous navigation using low throughput interfaces," in IEEE Int Conf Systems, Man, and Cybernetics (IEEE SMC 2011), 2011.

[22] R. Chavarriaga and J. Millán, "Learning from EEG error-related potentials in noninvasive brain-computer interfaces." IEEE Trans Neural Syst Rehabil Eng, vol. 18, no. 4, pp. 381-388, Aug 2010.

[23] R. Chavarriaga, A. Biasiucci, K. Förster, D. Roggen, G. Tröster, and J. d. R. Millán, "Adaptation of Hybrid Human-Computer Interaction Systems using EEG Error-Related Potentials," in 32nd Annual International Conference of the IEEE Engineering in Medicine and Biology Society (EMBC'10), 2010.

[24] S. Saeedi, R. Chavarriaga, M. C. Gastpar, and J. d. R. Millán, "Real-time Prediction of Fast and Slow Delivery of Mental Commands in a Motor Imagery BCI: An Entropy-based Approach," in IEEE Transactions on Systems, Man, and Cybernetics, vol. 42, no. 3, 2012, pp. 3327-3331.

[25] T. Carlson, R. Leeb, R. Chavarriaga, and J. d. R. Millán, "Online Modulation of the Level of Assistance in Shared Control Systems," in Proceedings of the IEEE International Conference on Sytems Man and Cybernetics (SMC 2012), 2012, pp. 3321-3326. 